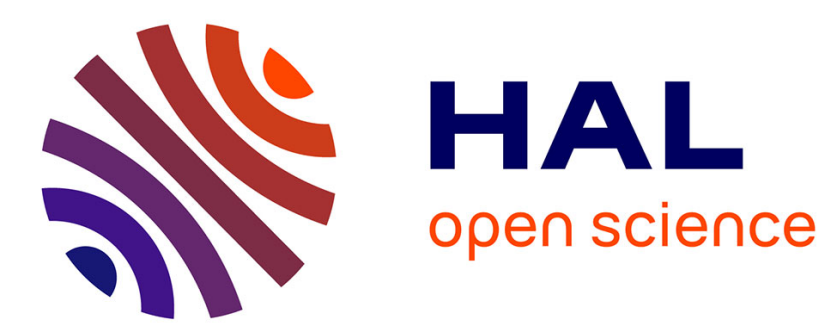

\title{
L'EMPLOI DES PLASTIQUES POUR L'EMBALLAGE DES PRODUITS LAITIERS
}

\author{
G. Genin
}

\section{To cite this version:}

G. Genin. L'EMPLOI DES PLASTIQUES POUR L'EMBALLAGE DES PRODUITS LAITIERS. Le Lait, 1963, 43 (428), pp.507-517. hal-00928341

\section{HAL Id: hal-00928341 \\ https://hal.science/hal-00928341}

Submitted on 1 Jan 1963

HAL is a multi-disciplinary open access archive for the deposit and dissemination of scientific research documents, whether they are published or not. The documents may come from teaching and research institutions in France or abroad, or from public or private research centers.
L'archive ouverte pluridisciplinaire HAL, est destinée au dépôt et à la diffusion de documents scientifiques de niveau recherche, publiés ou non, émanant des établissements d'enseignement et de recherche français ou étrangers, des laboratoires publics ou privés. 
Mogensen M. T. S., 1947. Appréciation du degré de protéolyse du fromage en prenant plus particulièrement en considération la formol titration (en danois). Meddelande fran statens Mejeriforsök, $\mathrm{n}^{\circ} 21$.

Pien J., Maurice G., 1937. Modifications dans la composition des fromages au cours de leur conservation prolongée, Le Lait, 17, 1040-1046.

Raibaud P., Herren R., Mocquot G., Kosikowski F. V., 1959. Mise en évidence dans certains fromages de systèmes enzymatiques actifs sur la tyrosine. Ann. Technol, agric., 8, 117-129,

Veisseyre R., Lenoir J., Marceron J. F. (Données non publiées).

Veisseyre R., Lenoir J., Pierre M. (Données non publiées).

\title{
L'EMPLOI DES PLASTIQUES POUR L'EMBALLAG DES PRODUITS LAITIERS
}

\author{
par \\ G. GENIN \\ Ingénieur E.P.C.I.
}

Depuis déjà plusieurs années, l'utilisation des plastiques s'est généralisée dans l'emballage et le conditionnement des produits alimentaires et en particulier des produits laitiers. L'emploi des matières plastiques dans l'emballage répond à des buts différents, soit simplement emballer les produits pour les conserver au frais et propres dans une pellicule se conservant elle-même convenablement, soit réaliser un véritable emballage étanche en vue par exemple de conserver des aliments congelés (dans ce cas la feuille d'emballage doit permettre ou non la "respiration du contenu»), soit assurer l'emballage de produits qui doivent ensuite, dans cet état, subir une cuisson, soit assurer la conservation et le conditionnement de produits liquides, soit enfin assurer le stockage de quantités importantes de produits dans de grands entrepôts.

On voit que les problèmes posés sont nombreux et il n'est donc pas étonnant que les solutions proposées soient différentes, en particulier depuis que l'industrie des produits alimentaires a à sa disposition une grande variété de matériaux plastiques. Dans cette étude, nous nous contenterons donc de décrire les pellicules plastiques utilisées dans l'emballage, énoncer leurs propriétés et leurs principales caractéristiques, quitte à revenir ultérieurement sur des formes plus particulières d'emploi des matériaux plastiques disponibles. 


\section{Polyéthylène}

On peut dire que le polyéthylène a été le premier thermoplastique utilisé sur une grande échelle par l'industrie de l'emballage et e'est par dizaine de milliers de tonnes que ce produit est aujourd'hui consommé sous différentes formes.

Les feuilles de polyéthylène se présentent sous l'aspect d'une pellicule solide, translucide, flexible, inodore et sans goût. Le polyéthylène n'est pas toxique et il résiste à l'action de la plupart des graisses, des acides gras et des huiles et il résiste parfaitement à l'eau. Il est perméable aux gaz, ce qui permet aux marchandises emballées de respirer. C'est un produit peu coûteux, qui peut se sceller à chaud à lui-même. Un film de polyéthylène est très élastique et peut subir un allongement de 600 p. 100 avant rupture. Le polyéthylène n'a pas de point de fusion bien défini, le début de fusion se produit vers $111^{\circ}$ et la fusion est complète à $115^{\circ}$, mais la résine utilisée pour la fabrication des pellicules a un point de fusion de l'ordre de $90^{\circ} \mathrm{C}$. Le tableau ci-dessous donne la perméabilité d'une feuille de polyéthylène, d'une épaisseur de $0,025 \mathrm{~mm}$, à la température de $25^{\circ}$, vis-à-vis de différents gaz et d'huiles essentielles.

Perméabilité par $\mathrm{m}^{2}$ et par jour

\begin{tabular}{|c|c|}
\hline Vapeur d'eau (air à $100 \%$ d'humidité) $\ldots .$. & $6,09 \mathrm{ml}$ \\
\hline 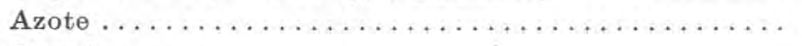 & $2,77 \mathrm{ml}$ \\
\hline Oxygène $\ldots \ldots \ldots \ldots \ldots \ldots \ldots \ldots \ldots \ldots \ldots$ & $8,47 \mathrm{ml}$ \\
\hline Hydrogène $\ldots \ldots \ldots \ldots \ldots \ldots \ldots \ldots \ldots \ldots \ldots \ldots$ & $30,0 \mathrm{ml}$ \\
\hline Gaz carbonique $\ldots \ldots \ldots \ldots \ldots \ldots \ldots \ldots \ldots$ & $44,6 \mathrm{ml}$ \\
\hline Essence de vanille $\ldots \ldots \ldots \ldots \ldots \ldots \ldots \ldots \ldots \ldots$ & $0,14 \mathrm{~g}$ \\
\hline Essence de muscade ........... & $57,5 \mathrm{~g}$ \\
\hline Essence de citron $\ldots \ldots \ldots \ldots \ldots \ldots \ldots \ldots \ldots \ldots \ldots$ & $109,6 \mathrm{~g}$ \\
\hline Essence de pamplemousse $\ldots \ldots \ldots$ & $129 \mathrm{~g}$ \\
\hline 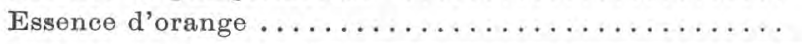 & $108 \mathrm{~g}$ \\
\hline
\end{tabular}

A la température ordinaire, certaines huiles ou graisses peuvent provoquer un gonflement et un changement de coloration du polyéthylène, mais en général les huiles végétales ou animales ont beaucoup moins d'action que les huiles minérales. Les pellicules peuvent être imprimées et scellées à chaud à une température de l'ordre de $120^{\circ}$.

Deux types de pellicules peuvent être utilisées : celles constituées uniquement de polyéthylène et les pellicules composites obtenues par l'assemblage de deux pellicules de matériaux différents: l'un étant destiné à renforcer les qualités mécaniques de la pellicule de polyéthylène et à modifier sa perméabilité. 


\section{Films de polyéthylène}

Ces films sont perméables aux gaz et ne conviennent pas pour l'emballage de produits à odeur prononcée ou pour l'emballage dans le vide.

Les produits desséchés comme le lait en poudre peuvent être emballés dans ces pellicules où ils se trouvent placés à l'abri de l'humidité. D'autre part, comme le polyéthylène est insensible à l'action des basses températures, ce produit convient à l'emballage de produits alimentaires congelés qui, en particulier, ne subissent pas de perte de poids par déshydratation dans les chambres froides.

\section{Films composites}

Pour augmenter les qualités des films de polyéthylène, on peut les associer à d'autres films. Par exemple, les films composites de polyéthylène et de cellophane peuvent être employés pour l'emballage dans le vide des produits alimentaires. On a également réalisé des feuilles de polyéthylène et de cellulose pour l'emballage de produits ayant subi une préparation par cuisson et qu'il suffit de réchauffer au moment de leur consommation en plongeant le sachet qui les contient dans l'eau bouillante. Les feuilles de polyéthylène renforcées par un papier fort ou par un carton sont également utilisées pour l'emballage d'un grand nombre de produits et nous citerons également leur association avec une feuille métallique, comme par exemple une feuille d'aluminium.

Une réalisation récente est constituée par les pellicules de polyéthylène ayant subi un double allongement suivant deux axes perpendiculaires, en même temps qu'un bombardement par des électrons. Le traitement s'effectue dans des installations spéciales dans lesquelles, en même temps que la pellicule est soumise à un effort de traction, elle est soumise à un rayonnement de haute énergie. Un film ainsi traité et porté ensuite à environ $90^{\circ}$ se rétracte d'environ 50 p. 100 de sa longueur initiale, mais ne subit plus ensuite, aucune variation de dimensions, quelles que soient les fluctuations de température et d'humidité. Ce film a une résistance à la traction de l'ordre de $7 \mathrm{~kg} / \mathrm{mm}^{2}$, c'est-à-dire 6 fois plus élevée que celle d'un film préparé avec des polyéthylènes basse densité courants, il est en outre parfaitement transparent et très brillant.

\section{Polypropylène}

Ce produit, dont l'apparition sur le marché remonte à 1957 , présente certains avantages sur le polyéthylène. Il est plus brillant et plus transparent, présente une plus grande résistance à la chaleur 
et de meilleures qualités mécaniques. C'est un produit bon marché dont la fabrication est en plein développement.

Incolore et sans goût, rigide et à grande dureté superficielle, c'est le plus léger des thermoplastiques courants $(d=0,905)$ et son point de fusion est de $165-170^{\circ} \mathrm{C}$. A $22^{\circ} \mathrm{C}$, sa résistance à la traction est de $5,30 \mathrm{~kg} / \mathrm{mm}^{2}$. Lorsque la température s'élève, ses qualités mécaniques diminuent, mais moins que celles du polyéthylène et on peut dire que jusqu'à $100^{\circ} \mathrm{C}$, il conserve ses propriétés et il est encore solide à la température de fusion du polyéthylène.

En ce qui concerne la perméabilité aux gaz, le polypropylène est supérieur au polyéthylène et le tableau 2 donne quelques indications déterminées sur un film de $0,025 \mathrm{~mm}$ d'épaisseur en 24 heures.

\begin{tabular}{|c|c|c|}
\hline & Film coulé & Film orienté \\
\hline 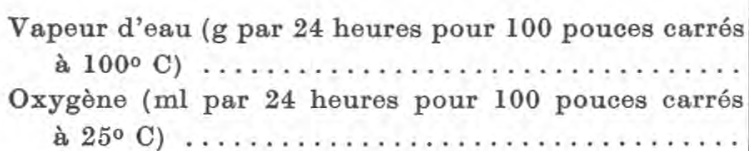 & $\begin{array}{l}0,76 \\
100\end{array}$ & $\begin{array}{l}0,25 \\
60\end{array}$ \\
\hline
\end{tabular}

Un sérieux inconvénient de ce produit par contre est qu'aux températures inférieures à $0^{\circ} \mathrm{C}$, il devient cassant et il ne peut donc être utilisé pour l'emballage des produits congelés. Cette difficulté peut être surmontée par un traitement d'orientation biaxiale qui rend le produit insensible aux basses températures, qui fait passer sa résistance à la traction de 3,50 à $5,60 \mathrm{~kg} / \mathrm{mm}^{2}$ et qui réduit pratiquement de moitié sa perméabilité aux gaz.

D'autres avantages de la pellicule de polypropylène sont sa résistance à l'abrasion, sa solidité, son insensibilité aux changements d'humidité, mais surtout, point déjà signalé, sa transparence.

Après un traitement superficiel convenable, on peut déposer sur le propylène des impressions en utilisant les mêmes encres que pour le polyéthylène.

Avec un produit dont l'apparition est relativement récente, il est difficile de faire la distinction entre les produits qui ont déjà atteint le stade commercial et ceux qui comme le polypropylène sont en cours d'expérimentation pratique et de prévoir dès maintenant quel sera l'avenir de ce produit. On peut cependant affirmer qu'il existe des débouchés certains pour le film de polypropylène, en particulier pour la qualité à allongement bi-axial, et sa fabrication est aujourd'hui entreprise dans un grand nombre de pays. 


\section{Polystyrène}

Depuis l'apparition du polystyrène qui coïncide avec l'avènement des caoutchoucs de butadiène-styrène, de nombreuses qualités de ce produit ont fait leur apparition, destinées aux industries de l'emballage; toutes se caractérisant par leur transparence, leurs qualités mécaniques et leur aspect. Une des caractéristiques les plus importantes de ce produit est sa facilité de moulage, la simplicité des opérations de formage des feuilles ou des plaques de polystyrène et par exemple, on tend de plus en plus, dans certains pays, à remplacer les gobelets en carton paraffiné par des gobelets en polystyrène dans les appareils distributeurs de boissons gazeuses.

Le polystyrène a trouvé trois groupes principaux d'applications dans l'emballage :

- Les pellicules ayant subi une orientation bi-axiale, utilisées pour l'emballage extérieur de paquets ou d'objets. La pellicule subit ensuite un chauffage qui la contracte sur la pièce emballée.

- Les feuilles plus épaisses qui, par exemple après formage dans le vide ou sous pression, peuvent servir à la confection de gobelets, de pots ou de récipients divers.

- La poudre à mouler, utilisée pour le moulage d'objets et de boîtes d'emballage.

\section{Pellicules de polystyrène}

Après orientation bi-axiale qui peut augmenter ses dimensions de 3 fois dans chaque sens, la pellicule obtenue est extrêmement solide, même en faible épaisseur, le produit est sans goût, inodore, non toxique et résiste aux graisses.

L'indice de réfraction du polymère lui confère un aspect cristallin et il transmet 90 p. 100 de la lumière. Il peut supporter des températures inférieures à $0^{\circ} \mathrm{C}$ et n'est pas déformé jusqu'à $70^{\circ} \mathrm{C}$ et plus. Voici d'ailleurs un extrait des propriétés d'une pellicule bi-orientée.

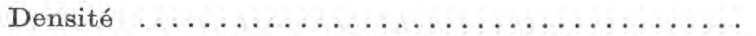

Transmission de la lumière $\ldots \ldots \ldots \ldots \ldots \ldots \ldots \ldots$

Absorption d'eau .....................

Résistance à la traction $\ldots \ldots \ldots \ldots \ldots \ldots \ldots \ldots$

Allongement . . . . . . . . . . . . . . . .

Résistance à la chaleur . . . . . . . . . . . . .

1,05
$92 \%$
$0,05 \%$

6,30 à $8,40 \mathrm{~kg} / \mathrm{mm}^{2}$

10 à $20 \%$ $78^{\circ} \mathrm{C}$

Le polystyrène résiste aux acides faibles, il n'est attaqué que par les acides oxydants, il résiste aux alcalis faibles et forts, il est soluble dans les hydrocarbures aromatiques et les hydrocarbures 
chlorés. Sa perméabilité à la vapeur d'eau est six fois supérieure à celle du polyéthylène et il convient donc pour l'emballage de denrées qui doivent respirer, puisqu'il permet le passage de la vapeur d'eau et de l'oxygène, tout en s'opposant à celui des huiles essentielles.

Le film bi-orienté présente de bonnes propriétés de vieillissement, par suite de sa faible absorption d'humidité et de l'absence de plastifiants. Il reste limpide, même aux basses températures, il est facilement utilisable en combinaison avec d'autres feuilles d'emballage, sous forme de laminé, par exemple avec le papier. Il peut être métallisé et les opérations d'impression sur film de polystyrène ne présentent aucune difficulté.

Les feuilles plus épaisses de polystyrène peuvent être facilement formées soit par le procédé par le vide, soit par emboutissage, soit par une combinaison de ces deux procédés. On opère dans ces cas sur des feuilles ou plaques dont l'épaisseur peut varier de 0,25 à 4,5 mm. On notera cependant que les objets ainsi obtenus, au contact des matières grasses contenues dans le lait, peuvent se fendiller.

Enfin le polystyrène peut être moulé facilement pour l'exécution de petits récipients destinés par exemple à contenir des portions d'aliments tels que crèmes glacées, fromage blanc, en vue de leur distribution dans les cantines ou les self-services. Dans ce domaine, les récipients en polystyrène moulé prennent de plus en plus la place du verre.

\section{Chlorure de polyvinylidène}

Sous le nom de Saran ou de Cryovac, on utilise des copolymères de chlorure de polyvinylidène et de chlorure de polyvinyle qui fournissent des pellicules dont les propriétés peuvent varier sensiblement suivant le degré de polymérisation et la composition du copolymère. Cette pellicule se contracte lorsqu'elle est chauffée à $70^{\circ}$ et peut donc être utilisée pour ce mode d'emballage particulier qui consiste à introduire l'objet à protéger dans un sac que l'on rétracte ensuite, afin qu'il épouse complètement la forme de l'objet.

La pellicule de chlorure de polyvinylidène présente une très faible perméabilité à l'oxygène et à d'autres gaz et constitue une barrière au passage de la vapeur d'eau. Elle est fréquemment employée, sous la forme de laminé, en combinaison avec d'autres pellicules.

Voici par exemple quelques propriétés de la pellicule vendue sous la marque Cryovac S : 
Limpidité

Epaisseur ..................

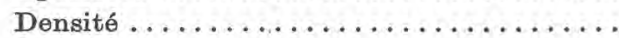

Résistance à la traction $\ldots \ldots \ldots \ldots \ldots$.

Allongement .................

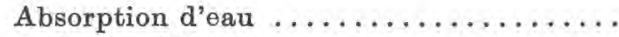

Point de fusion . . . . . . . . . . . .

Résistance aux rayons solaires ......... transparent ou opaque, au choix 0,020 à $0,075 \mathrm{~mm}$ 1,64 4,20 à $8,40 \mathrm{~kg} / \mathrm{mm}^{2}$ 75 à $120 \%$ négligeable en 24 heures $143^{\circ} \mathrm{C}$ bonne

En ce qui concerne son imperméabilité aux gaz et à la vapeur d'eau, voici quelques indications relatives à la pellicule de Saran :

Perméabilité à $25^{\circ}$ en $24 \mathrm{~h}$ par 100 pouces carrés

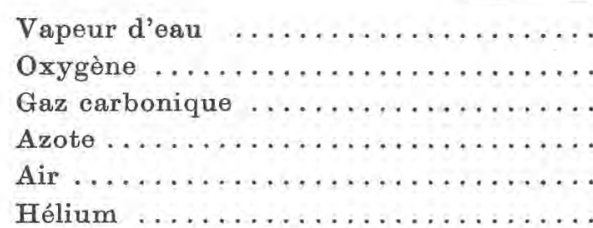

$<0,35 \mathrm{ml}$
$1 \mathrm{ml}$
3,8
0,16
0,21
40

Enfin, au point de vue chimique, le chlorure de polyvinylidène présente une excellente résistance aux acides minéraux dilués et concentrés (à l'exception des acides oxydants), aux acides organiques, aux alcalis (à l'exception de l'ammoniaque), aux hydrocarbures aliphatiques, aux huiles, aux graisses et aux cires, aux produits détersifs, il est gonflé ou dissous par les cétones cycliques et les éthers cycliques.

Dans certains pays, le Saran a trouvé d'importantes applications pour l'emballage des produits laitiers et particulièrement des fromages. Les fromages sans croûte peuvent par exemple être découpés en portions et emballés dans le Saran et conservés sur les étalages d'un magasin pendant deux semaines sans subir de perte de poids. Le film est complètement flexible, épouse la forme de la portion, d'autant plus qu'on peut contracter l'emballage de façon à éliminer totalement l'air et éviter toute formation de moisissure. On a également utilisé cette pellicule pour la conservation des fromages pendant une période de longue durée : 8 à 10 semaines, en évitant toute pénétration de l'oxygène et tout desséchement du produit. Un autre procédé consiste à déshydrater la surface de chaque portion par exposition aux rayons infra-rouges, jusqu'à ce que le produit se recouvre d'une couche huileuse. Ce traitement détruit également les bactéries et les portions peuvent alors être emballées dans un emballage unique sans risque de collage entre elles. 
Citons également pour l'emballage du fromage des sacs constitués d'une pellicule composite de Mylar (téréphtalate d'éthylèneglycol) de Saran et de polyéthylène, qui permet l'emballage dans le vide.

\section{Chlorhydrate de caoutchouc}

Le chlorhydrate de caoutchouc s'obtient en traitant une solution de caoutchoue dans un solvant organique par du gaz chlorhydrique. Après addition d'antioxydants et de plastifiants, Ia solution est coulée pour obtenir une pellicule. Le plastifiant est nécessaire pour obtenir un film souple et pliable, mais plus la proportion de plastifiant est élevée, plus le produit est mou et perméable aux gaz.

Ce produit est vendu sous la marque Pliofilm, c'est un produit transparent, extrêmement élastique, en particulier aux températures élevées et qui présente une imperméabilité suffisante pour pouvoir être utilisé dans l'emballage des liquides. Il peut être scellé à chaud, collé et employé sous forme de laminés avec d'autres feuilles : papier, feuilles plastiques ou feuilles métalliques. Il est livré en épaisseurs de 0,01 à $0,050 \mathrm{~mm}$ et en différents types, suivant la teneur en plastifiants. Voici par exemple quelques propriétés du type $\mathrm{N} l$ qui ne renferme qu'une partie de plastifiant et qui présente une faible perméabilité.

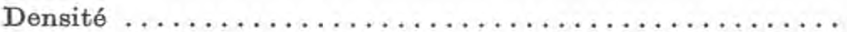

1,114

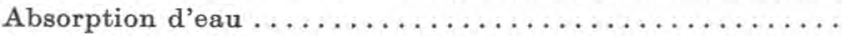

Température de scellement $\ldots \ldots \ldots \ldots \ldots \ldots \ldots \ldots \ldots$

Résistance aux basses températures $\ldots \ldots \ldots \ldots \ldots \ldots \ldots$

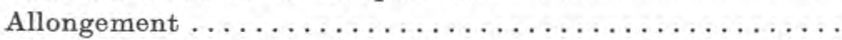

Résistance à la traction

nulle

$120^{\circ} \mathrm{C}$

$0^{\circ} \mathrm{C}$

$70 \%$

Résistance à la lumière solaire

$1,23 \mathrm{~kg} / \mathrm{mm}^{2}$

moyenne

Du fait de la grande variété de types de Pliofilm, cette pellicule peut être utilisée pour l'emballage d'un grand nombre de produits différents. Pour l'emballage de produits congelés, il est nécessaire d'employer les types très plastifiés. On l'emploie par exemple pour le doublage de sacs destinés à contenir du lait en poudre.

\section{Pellicules composites}

Les pellicules d'emballage que nous avons décrites précédemment ayant des caractéristiques différentes et qui se complètent parfois, il n'est pas étonnant que l'on ait cherché à combiner plusieurs pellicules différentes sous forme de laminés afin d'obtenir des pellicules composites présentant les caractéristiques désirées de résistance mécanique, d'aspect superficiel, d'imperméabilité, 
de rigidité etc. Pour ne citer qu'un exemple, la combinaison d'une pellicule de polyéthylène dont les propriétés d'imperméabilité aux gaz sont passables et d'une pellicule de Saran bien supérieure à ce point de vue, fournit un ensemble bénéficiant d'une bonne stabilité dimensionnelle et parfaitement imperméable. Evidemment, dans une telle combinaison, certaines difficultés peuvent apparaître principalement dans le collage ou la réunion des pellicules entre elles.

Nous avons donné en exemple, dans le tableau ci-dessous, quelques indications numériques sur la perméabilité de certaines de ces combinaisons.

\begin{tabular}{|c|c|c|}
\hline & $\begin{array}{c}\text { Oxygène } \\
100 \text { pouces carrés } \\
\text { par } 24 \mathrm{~h} \text {, sous } \\
1 \mathrm{~atm} .\end{array}$ & $\begin{array}{c}\text { Vapeur d'eau } \\
100 \text { pouces carrés } \\
\text { par } 24 \mathrm{~h} \text { à } 25^{\circ} \\
\text { (air à } 50 \mathrm{p} .100 \\
\text { d'humidité relative) }\end{array}$ \\
\hline Polyéthylène de $0,025 \mathrm{~mm} \ldots \ldots \ldots$ & $500 \mathrm{ml}$ & $0,24 \mathrm{~g}$ \\
\hline Cellophane de $0,025 \mathrm{~mm} \ldots \ldots \ldots \ldots$ & 2,4 & 0,23 \\
\hline Saran de $0,0025 \mathrm{~mm} \quad \ldots \ldots \ldots \ldots \ldots$ & 0,5 & 0,07 \\
\hline Cellophane de $0,025+$ Saran de & & \\
\hline $0,0025+$ polyéthylène de $0,050 \mathrm{~mm}$ & 0,5 & 0,02 \\
\hline Cellophane de $0,025+$ Saran de & & \\
\hline $0,0025 \mathrm{~mm} \ldots \ldots \ldots \ldots \ldots \ldots \ldots$ & 0,2 & 0,02 \\
\hline $\begin{array}{r}\text { Cellophane de } 0,025+\text { polyéthylène } \\
\text { de } 0,050 \mathrm{~mm} \ldots \ldots \ldots \ldots \ldots \ldots \ldots\end{array}$ & 2,9 & 0,08 \\
\hline Polyéthylène de $0,050+$ Saran de & & \\
\hline $0,0025 \mathrm{~mm} \ldots \ldots \ldots \ldots \ldots \ldots$ & 0,6 & 0,04 \\
\hline
\end{tabular}

On dispose de deux méthodes pour réaliser l'assemblage de pellicules. Dans la première, on boudine dans un extrudeur une couche de plastique sur une feuille support fabriquée à l'avance. De cette façon, on réalise un véritable contact moléculaire. Par exemple, le polyéthylène peut être facilement laminé de cette façon sur d'autres supports. Dans la seconde méthode, des pellicules préparées à l'avance sont collées l'une à l'autre en utilisant généralement des adhésifs synthétiques. Ce procédé est moins satisfaisant.

Quel que soit le procédé adopté, les deux constituants doivent être compatibles, et se comporter de la même façon vis-à-vis des facteurs extérieurs pour ne pas risquer de décollements ou de formation de rides.

Parmi les différents types de pellicules composites, le groupe le plus important est constitué par les pellicules de cellulose régénérée 
(Cellophane), produit qui n'est pas très imperméable à la vapeur d'eau et de polyéthylène. On peut également laminer avec la Cellophane du Saran (sur les deux faces) et obtenir un produit présentant une haute stabilité dimensionnelle, brillant, scellable à chaud et résistant à la lumière ultra-violette et ajouter encore une pellicule de polyéthylène pour augmenter la résistance au déchirement. On peut également coller deux feuilles de Cellophane avec une couche intermédiaire de cire, l'une des feuilles de Cellophane pouvant être revêtue d'une pellicule de plastique.

Le papier est également fréquemment enduit de cire ou de plastique, ce qui le rend collable à chaud (procédé par enduction) ou revêtu, avec l'aide d'un adhésif, d'une pellicule plastique ou métallique. Un exemple est donné par les feuilles composites de papier et de feuille d'aluminium. Ces feuilles métalliques peuvent être également collées à des feuilles plastiques, en particulier de polyester (Mylar). Un tel film composite se caractérise par ses qualités de scellement et on peut encore augmenter son imperméabilité aux gaz et aux vapeurs en le combinant à une pellicule de polyéthylène.

Enfin, nous avons donné dans le tableau précédent quelques exemples de combinaison de pellicule plastique, auxquels nous ajouterons les pellicules constituées par une feuille de polyéthylène et de polyester qui bénéficie de l'imperméabilité du polyéthylène et de la stabilité aux hautes températures du polyester.

Parmi les applications de ces fibres composites qui intéressent l'industrie des produits laitiers, nous citerons les suivantes. Si pour un emballage destiné à n'assurer qu'un magasinage de quelques jours des portions de fromage, une feuille de Cellophane, de Saran ou de Pliofilm peut suffire, on utilise un emballage composite de Saran et de Cellophane pour un stockage un peu plus prolongé et même une feuille triple de polyéthylène, de Cellophane et de Pliofilm pour une conservation de longue durée. Des études sont actuellement en cours sur les films de polyester, de Saran et de polyéthylène.

Si le beurre est couramment enveloppé dans de simples feuilles de papier parchemin, cet emballage n'est pas suffisamment imperméable à l'oxygène et à la lumière pour prévenir l'altération du produit par rancissement. De meilleurs résultats sont obtenus par une combinaison de papier parchemin et de feuille d'aluminium, ou de Cellophane et de papier parchemin.

Théoriquement, la grande diversité des différentes pellicules d'emballage dont on dispose aujourd'hui et le grand nombre de leurs combinaisons possibles devraient permettre de satisfaire à toutes les exigences des utilisateurs. En fait, l'apparition sur le 
marché de produits alimentaires nouveaux et bénéficiant d'une présentation nouvelle et le désir d'a méliorer la présentation, obligent les fabricants de pellicules d'emballage à rechercher sans cesse des produits ou des combinaisons nouveaux. L'industrie laitière, comme les autres industries alimentaires, ont donc la possibilité de choisir, parmi la grande diversité de produits mis à leur disposition, ceux qui conviennent le mieux à la nature des marchandises emballées, aux conditions de magasinage imposées et aux désirs de la clientèle.

\section{Bulletin analytique}

\section{(Revues)}

\section{Acide lactique et lactates}

\section{Trifonov (A.) et Vitanov (T.). - Recherches sur le complexe} ferrique formé avec l'acide lactique en solution aqueuse. Izvest. Khim. Inst. Bulgar. Akad. Nauk, 1960, t. VII, p. 309.

La formation de complexes de fer trivalent avec l'acide lactique dépend de la concentration de l'acide lactique et du $p H$. On a étudié photométriquement le complexe formé pour une concentration constante en ion ferrique, une concentration variable en acide lactique, pour un $p \mathrm{H}$ de 1,9 à 2 et pour une longueur d'onde de $405 \mathrm{~m} \mu$. Les complexes formés répondent aux formules $\mathrm{FeOOCCHOHCH} \mathrm{H}_{3}++(\mathrm{I}), \mathrm{Fe}\left(\mathrm{OOCCHOHCH}_{3}\right)_{2}+$ (II) et $\mathrm{Fe}\left(\mathrm{OOCCH} \mathrm{OCH}_{3}\right)_{3}$ (III). On a déterminé la constante de dissociation de ces produits soit par le calcul, soit expérimentalement.

\section{Prior (J. T.), Cronk (G. A.) et Ziegler (D. D.). - Modifications} pathologiques associées à l'inhalation du lactate de zircomium-sodium. Arch. Environmental Health, 1960, t. I, p. 297.

Des lapins exposés à une atmosphère contenant $0,049 \mathrm{mg}$ par $\mathrm{ml}$ de $\mathrm{NaH}_{3}\left[\mathrm{ZrO}\left(\mathrm{OCHCH}_{3} \mathrm{COO}\right)_{3}\right]$ à des intervalles de 5 minutes pendant 20 minutes, ne présentent aucune irritation. Ce traitement a été poursuivi pendant 6 semaines et l'autopsie du corps des animaux a montré la présence de nodules jaunes dans les lobes supérieurs des poumons. Dans certains animaux, on a également observé des adhésions plurales, un phénomène de congestion des sinus nasaux et des amas granulaires noirs dans le foie.

\section{Grimm (A.I.).-Emploi d'antiseptiques et d'antibiotiques pour}

le stockage des carottes. Sbornik Nauch. Rabot, Leningrad.

Inst. Sovet. Torgovli im F. Engel'sa, 1959, n ${ }^{\circ} 15$, p. 17.

On a étudié l'influence antiseptique d'un certain nombre de substances telles que solution de borax à 1 et à 3 p. 100, acide borique à 0,5 p. 100 , 